\title{
Method of Geometric Connected Disk Cover Problem for UAV realy network deployment
}

\author{
Liu Chuang, Lv Na, Chen Ke-fan, Zhang Bu-shuo,Cao Fang-bo \\ School of informational and navigation, Air Force Engineering university, Xi'an 710077.China
}

\begin{abstract}
Aiming at the problem of the effective connectivity of a large number of mobile combat units in the future aeronautic swarm operation, this paper proposes an idea of using UAV(Unmanned Aerial Vehicle) to build, and studies the deployment of the network. User coverage and network connectivity are important for a relay network planning which are studied separately in traditional ways. In order to effectively combine these two factors while the network's survivability is taken into account. Firstly, the concept of node aggregation degree is proposed. Secondly, a performance evaluation parameter for UAV relay network is proposed based on node aggregation degree, then analyzes the lack of deterministic deployment and presents one a PSO (VFA-PSO) deployment algorithm based on virtual force. Finally, compared with the existing algorithms, the validity and stability of the algorithm are verified. The experimental results show that the VFA-PSO algorithm can effectively improve the network coverage and the survivability of the network under the premise of ensuring the network connectivity, and has better deployment effect.
\end{abstract}

Keywords—aeronautic swarm; unmanned aerial vehicle relay; network deployment; invulnerability; particle swarm optimization;

\section{INTRODUCTION}

With the deepening of the digital battlefield, a small number of combat platform to complete a single combat mission combat mode has been unable to meet the complex and changing battlefield environment. In order to make the air combat platform collaborative and efficient completion of operations, integrated system to realize multi platform, multi task combat, researchers will biological swarm concept applied to the field of air combat and put forward the concept of aeronautic swarm [1 3]. There are usually many types of combat platforms in aeronautic swarm, such as early warning aircraft, fighters, reconnaissance aircraft, unmanned aerial vehicles, etc., and the scale is large, generally reach dozens, hundreds, and platform mobility, wide distribution. In order to solve the different operational tasks of OODA (OberveOrient - Decide-Attack), the different types of platforms are based on the dynamic combination of task requirements, and the OODA complete combat process is realized on a continuous, continuous and closed loop basis on the basis of the coordinated operation of OODA .

In the aeronautic swarm combat environment, the platform position changes with the target location, the platform combination with the task requirements change, so that the distribution of the aeronautic swarm platform is random, discrete and wide area characteristics. The distribution of the platform matches the operational and operational objectives, but may not match the communication topology, that is, some platforms may become isolated nodes of the communication, resulting in the information can not be transmitted to each other, the swarm collaborative task can not be achieved. Therefore, how to achieve a large number of mobile aircraft in the mobile, decentralized combat units between the effective connectivity is the need to study the problem. The use of
UAV relay can effectively solve the above problems. UAV relay platform with a wide range of coverage, easy deployment, the use of flexible features, and can achieve over-the-horizon communication [4].

In this paper, we present a UAV relay network to support aeronautic swarm operations, and study the deployment of UAV relay network. This paper is organized as follows. In paper Section II we review related work .In Section III we formulate the problem, proposed the node aggregation degree and the deployment evaluation function. A random deployment algorithm based on particle swarm is designed in Section IV. In Section V we evaluate the algorithms via simulation. We summarize the results in Section VI.

\section{RELATED WORK}

The problem of network deployment is a pre-planning problem of the network. At present, the related research on UAV relay network is divided into two categories. One is the dynamic maintenance of UAVs. This kind of research mainly focuses on the low speed users to study the optimal flight path and network performance optimization of relay UAV[5-6]. Because this paper mainly focuses on the background of aviation cluster operations, UAVs have lower speeds compared to combat aircraft, can not be dynamically maintained. Therefore, this paper studies relatively static network deployment. This type of research is divided into deterministic deployments [7] and random deployment [8].

Deterministic deployment refers to the fact that the nodes are placed at the specified location according to a certain geometrical structure in order to obtain the minimum or optimal connectivity and coverage of the nodes. The deterministic deployment approach is applicable to situations 
where the environment is in good condition and the fixedpoint deployment costs are low. The advantage is that the number, density, location or proximity of the nodes in the network can be calculated and arranged according to different connectivity radius and coverage radius to obtain the optimal network deployment effect with the least number of nodes or the connectivity coverage. The disadvantage is that there is a boundary area resource redundancy, flexibility is poor, does not apply to irregular areas.

The location information of the nodes in the random deployment is unpredictable. The optimization problem is constructed according to the known conditions, and the location points of the deployment are obtained by intelligent algorithms such as genetic, virtual force and particle swarm. It has strong flexibility and more practicality. At present, the study of coverage and connectivity problems is usually independent of them to optimize, how to combine the two is the focus of many scholars. [9] designed a greedy algorithm, the algorithm first to complete the area coverage, in the realization of network connectivity, and finally delete the redundant deployment node to amend. However, there is a certain blindness in the greedy algorithm, and separate consideration and coverage will also affect the final outcome. In this method, the nodes are initially moved to the center of the region by gravity, and when the nodes are gathered to a certain extent, the network is connected, and the nodes are uniformly spread through the repulsive force to realize the maximum coverage of the nodes. However, this method is easy to cause nodes to always converge in the central part of the region, can not effectively achieve the edge of the area near the coverage. The use of particle swarm optimization algorithm for network deployment usually assumes that the network is connected, in the case of a large number of nodes. Does not consider the number of nodes in the case of limited coverage and connectivity to deploy.

\section{SCENES AND MODELS}

\section{A. Scene Description}

The discussion of this paper is based on the swarm combat application scenario shown in Figure 1, where a number of types of combat aircraft are scatred, with single isolated aircraft nodes and formation subnets, which are dynamically organized according to different combat missions. The distribution of these aeronautical swarm platforms presents randomness, discretization and wide-area characteristics. Therefore, this paper transforms the effective connectivity problem of decentralized maneuvering unit into the coverage problem of task area. The task area is divided according to the specific combat missions and scheduled routes in advance, can be a regular task area, it can be irregular. Through the rational deployment of multiple trunk UAVto achieve the stability of the task area coverage for the entire operational process to provide communication support.

\section{B. The geometric model of relaying unmanned aerial vehicles}

In general, the coverage area of the relay UAV is determined by the altitude of the flight, the height of the coverage area and the maximum propagation distance of the relay signal, and the maximum propagation distance of the relay signal is related to the status of the relay link channel. In this paper, considering the relay of the combat aircraft, assuming that the maximum propagation distance of the relay signal is $\mathrm{R}$, the deployment height of the relay unmanned aerial vehicle is $\mathrm{H}$, and for the aerial area with height $h$, if the transmission power and the antenna beam limitation factor are not taken into account, The maximum coverage radius of the relay signal is

$$
r(h)=\sqrt{R^{2}-(H-h)^{2}}
$$

As the height difference between the different air platforms is tens of meters to several kilometers, and the maximum transmission distance of the relay signal can reach hundreds of kilometers or more, the height difference between the platforms and the horizontal distance between each other can be almost ignore. Therefore, in order to facilitate modeling, this paper assumes that the relay UAV has the following characteristics:

(1)on the task area of the coverage radius and relay UAV connected between the same radius, are set to R;

(2) Consider deployment issues on a two-dimensional plane;

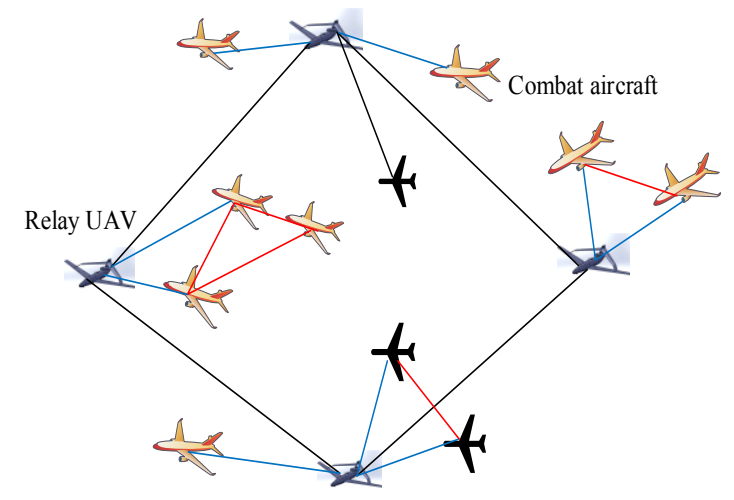

Figure.1. Schematic diagram of the scene

\section{RELATED DEFINITIONS}

\section{Definition 1. The degree of aggregation}

For a network, the invulnerability of the network is to measure the ability of the network topology to remain connected when the attack fails. At present, the survivability optimization model focused on topology optimization, mainly discusses two problems: to meet the requirements of network connectivity, the lowest total cost or at a given cost, the topology optimization design of the largest network connectivity. In this paper the UAV relay network deployment issues, as the relay UAV number, radius of communication is limited, how to construct a under this condition should not only satisfy the coverage and connectivity requirements, at the same time, the largest network of survivable deployment mode is the main problem considered in this paper. 
In a relay network, each relay node not only wants to relay users within its own coverage area, but also consider forwarding information for neighbor nodes. Therefore, according to the problem of unmanned aerial vehicle relay network deployment, combined with the degree of cohesion and the function of the relay node itself, the evaluation parameter of the node aggregation network resource is defined, that is, the aggregation degree $\beta_{i}$ of the relay node $\mathrm{i}$ :

$$
\beta_{i}=t_{1} \frac{n-1}{2 \sum_{i, j \in V} d_{i j}}+t_{2} \frac{\sum N_{i}(S)-\sum_{n \neq i}(S)}{N_{0}(S)}
$$

In order to evaluate the network coverage performance effectively, this paper uses the grid method to divide the task area into grid. Assume that the number of pixels in the task area is $m \times n$. The area coverage ratio $R_{\text {area }}(S)$ of the node set $\mathrm{S}$ is the ratio of the sum of the total number of pixel points of the node set $\mathrm{S}$ to the total pixel of the task area. Where $\mathrm{n}$ is the number of nodes in the network, $d_{i j}$ is the shortest distance between any two points $i$ and $j$ in the network, and $V$ is the set of all the nodes in the network. $N_{0}(S)$ is the total number of pixels that a single node covers. And $t_{1}, t_{2}$ is the weight coefficient, in order to balance effect of network topology and coverage changes on the degree of aggregation.

\section{DESIGN OF DEPLOYMENT ALGORITHM FOR UAV RELAY NETWORK}

At present, there are two characteristics of the research on connectivity and coverage problems by means of intelligent algorithms such as genetic, virtual force and particle swarm. (1)There are fixed nodes and mobile nodes in the network, and the location of the mobile nodes is optimized by the intelligent algorithm. (2) The connectivity radius of the node is much larger than the coverage radius of the node, and the number of nodes is relatively dense. The above characteristics make the study of network deployment problems usually based on the default network is connected to optimize. In the context of this paper, there is no fixed node, and the task area is large, the number of relay unmanned aerial vehicles, the communication radius is limited, the particle search results are prone to the interconnection between the network nodes can not judge the quality of the solution, To a certain extent limit the use of PSO algorithm.

\section{A. Deterministic deployment model analysis}

Strip, triangular, quadrilateral, hexagonal, and so on because of the simple deployment of the deployment of wireless sensor network deployment and terrestrial emergency wireless communication relay network deployment issues. There are a lot of literature from the perspective of coverage and connectivity of these four models to study, so do not do too much. In this paper, based on the coverage of connectivity, the aggregation degree of nodes is analyzed. The deployment mode of several existing deployment modes is analyzed and the deployment mode satisfying the requirements of this paper is studied.

Figure 3 shows the four deployment modes that the size of the mission area is $500 \mathrm{~km} \times 400 \mathrm{~km}$ and the communication radius $\mathrm{R}$ is $100 \mathrm{~km}$. Table $\mathrm{I}$ gives a performance comparison of the four deployment patterns.

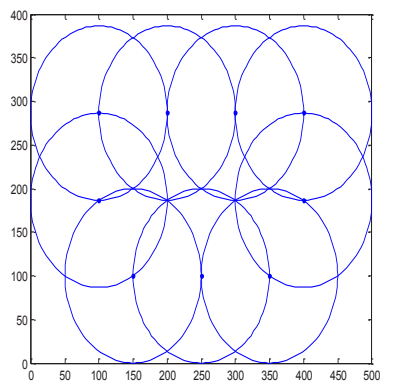

a. Strip deployment model

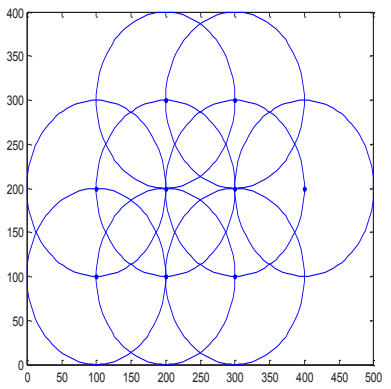

c. Quadrilateral deployment model

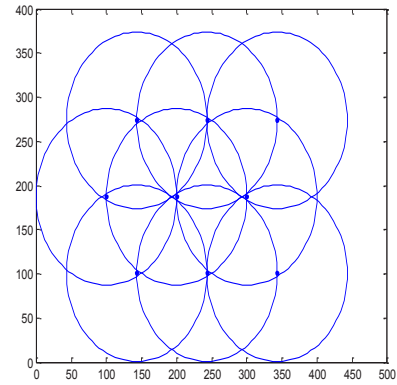

b. Triangular deployment model

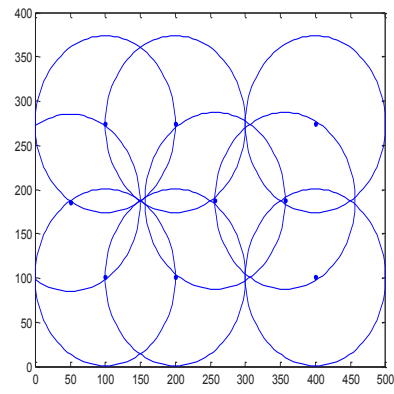

d. Hexagonal deployment model
Figure 3. schematic diagram of four deterministic deployment mode

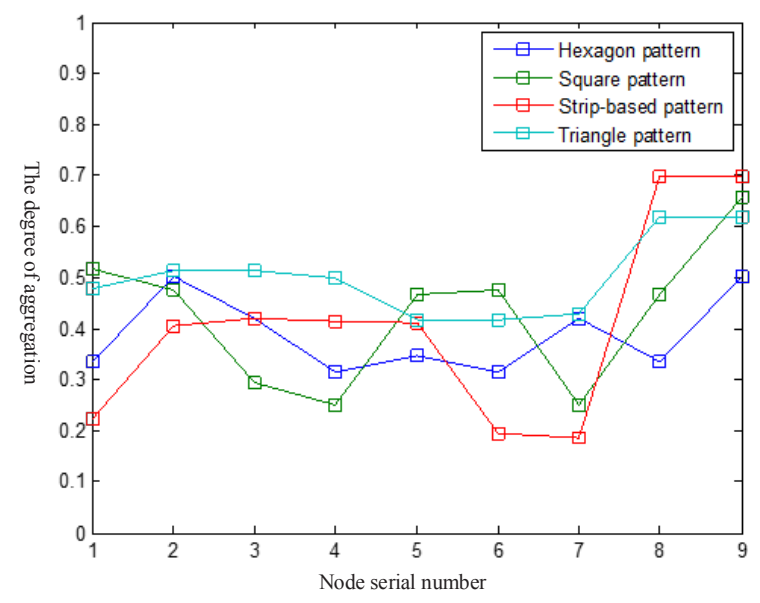

Figure 4 . The diagram of the variation of the node aggregation degree

TABLE I. THE PERFORMANCE COMPARISON OF THE FOUR DEPLOYMENT PATTERNS

\begin{tabular}{cccc}
\hline PATTERNS & COVERAGE RATE & AVERAGE DEGREE & VARIANCE \\
\hline STRIP & $84.60 \%$ & 0.3780 & 0.0057 \\
\hline TRIANGULAR & $70.91 \%$ & 0.5022 & 0.0060 \\
\hline QUADRILATERAL & $76.70 \%$ & 0.4278 & 0.0186 \\
\hline HEXAGONAL & $83.12 \%$ & 0.4051 & 0.0371 \\
\hline
\end{tabular}


It can be seen from Table I that in the same number of relay nodes, the deployment coverage ratio is the highest. Contrast stripe pattern and hexagonal pattern can be seen that although both coverage are smaller, but the hexagon mode node concentration variance is bigger, each node in the network "importance" gap is bigger, easy to cause this topology network anti-destroying ability is poor. And the pattern of stripe, although the node is distributed more evenly, and the coverage is higher, but the highest connection degree of the node is 2 , the network robustness is poor, unsuitable for practical application. Triangle and quadrilateral model can provide a better for network connectivity, but the triangle pattern redundant nodes is bigger, the coverage is low, can lead to a number of relay nodes use too much, not suitable for limited number of relay UAV communication relay network deployment.

Result in practical application, the task area will become irregular shape, fixed deployment model can produce covered hole in the region boundary, the ground base station or emergency relay network is mostly through the border region put node to "fix" connect the weak area of coverage. Such repairs, while filling the gaps in the cover, will actually cause the area near the border to be repeatedly covered, causing considerable redundancy. This paper proposes an algorithm for boundary region deployment - based on improved particle swarm algorithm.

\section{B. PSO algorithm principle}

Particle Swarm Optimization (PSO) is a new evolutionary computing technology based on group intelligence, which is widely used in various optimization fields. Each particle in the PSO algorithm represents a potential optimal solution, and each particle perturbs its flight trajectory according to its own current information, its own historical information and population information, so as to find the optimal solution in the search space. Let the population of $\mathrm{m}$ particles search the Q-dimensional space (ie, the dimension of each particle). The position of particle $i$ is expressed as $X_{i}=\left(x_{i 1}, x_{i 2}, \ldots x_{i Q}\right)$. The velocity represented by particle $i$ is expressed as $V_{i}=\left(v_{i 1}, v_{i 2}, \ldots v_{i Q}\right)$. The basic PSO algorithm is updated as follows:

$$
\begin{aligned}
v_{i Q}(k+1)= & w \times v_{i Q}(k)+c_{1} \times \gamma_{1} \times\left[p(k)-x_{i Q}(k)\right] \\
& +c_{2} \times \gamma_{2} \times\left[g(k)-x_{i Q}(k)\right]
\end{aligned}
$$

The location update formula for the particle in the search process is:

$$
x_{i Q}(k+1)=x_{i Q}(k)+v_{i Q}(k+1)
$$

In the formulas (4) and (5), $k$ represents the number of iterations; $w$ is the inertia weighting factor; $c_{1}$ and $c_{2}$ are the learning factor; $\gamma_{1}$ and $\gamma_{2}$ are the random number within the $(0,1)$ interval; $v(k) \in\left[-V_{\min }, V_{\max }\right]$ represents the velocity range of the particle; $x(k) \in\left[L_{\min }, L_{\max }\right]$ represents the search space; $p(k)$ is the historical value of the particle itself; $g(k)$ is the historical optimal value of the whole population;
Particle velocity and position update can directly determine the quality of the solution and the efficiency of the solution. Although the update of the particle position is too fast, the particle will jump out of the local optimal solution to a certain extent. However, due to the limitation of the connectivity between the relay nodes, the destination location update will cause the relay nodes to not communicate with each other. In view of this, in order to consider the network coverage and connectivity to deploy the node, and to avoid the situation can not calculate the value of the situation because the node is not directly connected, adding virtual force to affect the location of the node update after each iteration.

In a two-dimensional plane, if the particle swarm algorithm is used to deploy the $\mathrm{N}$ relay nodes, the search space dimension of the algorithm is $Q=2 \mathrm{~N}$. In the process of particle motion, the quality of the solution depends on the fitness function. In this paper, the reciprocal of the deployment evaluation function is taken as the fitness function Fitness of the PSO algorithm. According to the above rules, design PSO deployment algorithm.

\section{SIMULATION ANALYSIS}

In a clocked at $3 \mathrm{GHZ}$, the memory is $4 \mathrm{~GB}$ on the PC, the proposed algorithm is validated based on the MATLAB environment. The area of the task area is set $500 \mathrm{~km} \times 400 \mathrm{~km}$ so that the communication radius $\mathrm{R}$ of the relay unmanned aerial vehicle is $120 \mathrm{~km}$. Learning factors $c_{1}$ and $c_{2}$ are both 2. $w_{\text {max }}=0.9, \quad w_{\text {min }}=0.4$ and $\left|F_{t h}\right|=5, \quad c_{3}=0.5, \quad w_{\alpha}=5$ and $w_{r}=0.2$, Maximum displacement distance $\max$ step $=30 \mathrm{~km}$.

In order to compare the performance of VFA-PSO algorithm, the basic PSO algorithm and two improved PSO algorithms are used in the simulation experiment. One is based on the speed update factor improved with gravitational and repulsive PSO (combined gravitational and repulsion PSO, CAR-PSO) algorithm. Another PSO (explorative capability enhancement PSO, ECE-PSO) algorithm is based on improved particle search capabilities. The group size is set to 10 and the maximum number of iterations is 100 . For these three algorithms in the iterative process, due to the connection between nodes can not calculate the fitness value, we do not take the particle fitness value comparison.

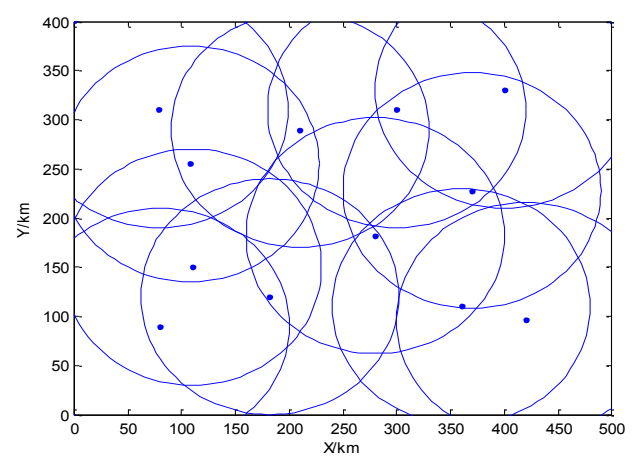

Figure .5. VFA-PSO algorithm deployment diagram 


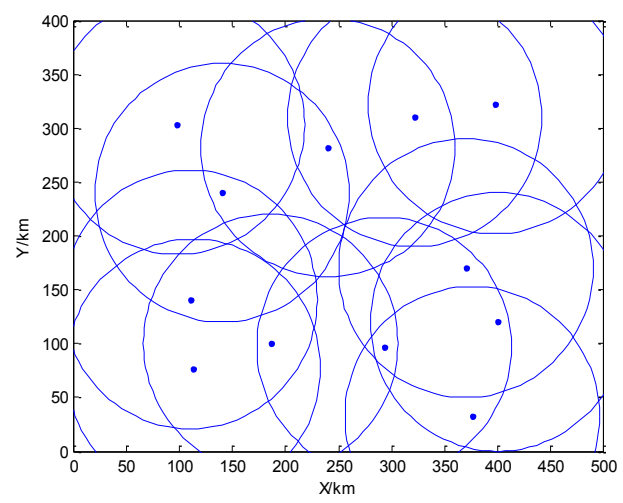

Figure.6. CRC-PSO algorithm deployment diagram

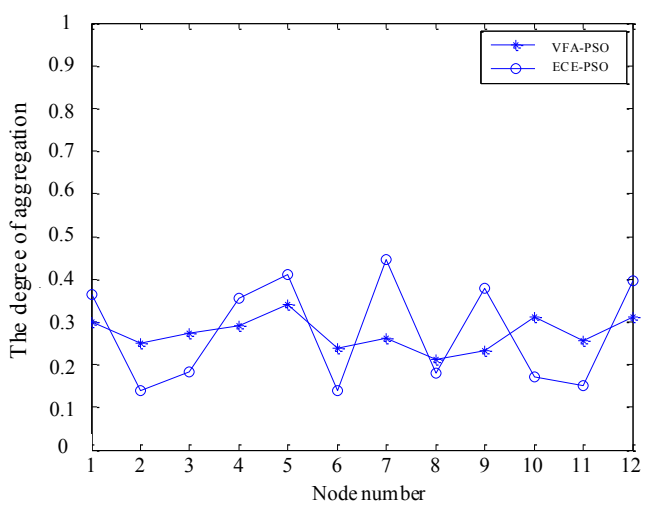

Figure.7. The curve of node aggregation degree change in two deployment situations

First, in order to verify the effectiveness of node aggregation degree for network survivability evaluation, 12 nodes are deployed in the task area. Figure 5 shows the results of the VFA-PSO algorithm deployment with a coverage of $98.7 \%$. Figure 6 shows the coverage of the CAR-PSO algorithm. The coverage rate is $97.3 \%$. Figure 7 shows the node aggregation degree of the two deployment results. The coordinates of the nodes are sorted according to the $\mathrm{x}$-axis coordinates from small to large. However, as can be seen from Figure 7, the variance of the network node aggregation in Figure 4 is significantly smaller than that of Figure 6. From the perspective of the survivability of the network, we can see that, for example, the number of nodes with the corresponding coordinates $(290,97)$ in Figure 6 is the largest, and the node is removed. The coverage does not change, but the network will not be connected. In the deployment result of Fig. 1 (b), the node distribution is more "scattered". It can be clearly seen in Figure 4 and Figure 6 that each node in the deployment network of Figure 6 has a large gap in "action", resulting in poor distribution of nodes with uneven distribution of nodes. This verifies the accuracy of the node aggregation degree definition and can effectively evaluate the role of each node in the relay network.

\section{CONCLUSIONS}

In this paper, we puts forward an idea of using UAV to construct relay network and study the deployment of the network based on the background of aeronautic swarm operations. In order to consider the deployment of combat unit coverage, network connectivity and survivability, the concept of node aggregation degree is put forward under the constraints of the number of relay UAV and the communication radius. And designs a VFA-PSO deployment algorithm based on virtual force adjustment. The simulation results show that the VFA-PSO algorithm can effectively improve the network coverage and the survivability of the network under the premise of ensuring the network connectivity, and has better deployment effect.

\section{ACKNOWLEDGMENT}

This work was supported by the National Natural Science Foundation of China under Grant No.61472443 and Research on the Application of Swarm Intelligent Induced Emergence in Aeronautic Swarm.

\section{REFERENCES}

[1] Duan H B, Shao S, Su B W. New development thoughts on the bioinspired intelligence based control for unmanned aircraft system flight plan.USA:Washington DC: 2009-2047[R].2010.

[2] Huo D J.Operational of network swarms [M].Beijing : National Defense University Press, 2013.

[3] Chaumette S, Laplace R, Mazel C, et al. CARUS, an operational retasking application for a swarm of autonomous UAVs: First return on experience[C]// Military Communications Conference, 2011 Milcom. IEEE, 2012:2003-2010.

[4] Duan X H, Wang J W, Zhao Y P, et al. Design and Implementation of UAV in Communication Relay[J]. Sci-tech Innovation and Productivity, 2016.

[5] Marinho M A M, Freitas E P D, Costa J P C L D, et al. Using cooperative MIMO techniques and UAV relay networks to support connectivity in sparse Wireless Sensor Networks[C]// International Conference on Computing, Management and Telecommunications. IEEE, 2013:49-54.

[6] Journal of Tsinghua University (Science and Technology), 2011 (2): 150-155 .Department of Computer Science and Technology, Tsinghua University, Beijing 100084, China); A Multi-UAV Relay Network Supporting Mobile Ad Hoc Networks [J].

[7] Khoufi I, Minet P, Laouiti A, et al. Survey of Deployment Algorithms in Wireless Sensor Networks: Coverage and Connectivity Issues and Challenges $[\mathrm{J}]$. International Journal of Autonomous \& Adaptive Communications Systems, 2014.

[8] Zhou F B, Xue S L. Advanced Sequence-Based Localization Algorithm in Wireless Sensor Networks[J]. Applied Mechanics \& Materials, 2014, 651-653(1):387-390.

Liu Chuang is a Master student at Air Force Engineering university, He received his bachelor of engineering in Chongqing University. His research interests are on the topics of Military aviation communication.

Lv $\mathrm{Na}$ is a Professor, doctoral supervisor at Air Force Engineering university, His research interests are on the topics of Military aviation communication.

Shaanxi Province, Xi'an Lianhu District Feng Gao Road No. 1, Phone number 13289396256, 